\title{
El Patrimonio Marítimo, la importancia de su Conservación y Uso Turístico
}

\author{
Lic. Ernesto Piero BAZZETTI DE LOS SANTOS ${ }^{7}$
}

\begin{abstract}
RESUMEN:
Esencialmente trataré de ofrecer una visión general sobre la importancia patrimonio marítimo. No pretendo llevar a cabo una exhaustiva descripción temática sino más bien indicar las distintas perspectivas que se tienen y los principales actores que intervienen en las mismas. Este trabajo se centrará básicamente el entender esta clasificación en el marco concreto del patrimonio, con la finalidad de dar inicio a una secuencia de artículos que incluirá otros elementos del patrimonio marítimo como, museos marítimos, buques museos, entre otros.
\end{abstract}

PALABRAS CLAVE: Patrimonio, Arqueología, Museo, Comunidad, Conservación, Turismo.

\section{ABSTRACT:}

Essentially I will try to provide an overview of the maritime heritage importance. I do not intend to make a thorough thematic description but rather indicate the different perspectives are taken and the main actors involved in them. This work will be basically focus in understand this classification in the specific context of heritage, in order to kick off the sequence of items to include other elements of maritime heritage as maritime museums, museum ships, among other.

KEYWORDS: Heritage, Archeology, Museum, Community, Conservation, Tourism.

Junto al patrimonio cultural, patrimonio artístico, patrimonio arquitectónico, etc., ha surgido en los últimos años una nueva categoría: el patrimonio marítimo. Bajo este calificativo se agrupan todos aquellos exponentes y manifestaciones de las técnicas, cultura y tradiciones marineras que la humanidad ha generado a lo largo de los siglos así como el medio natural en donde estas se desarrollan. Esto es importante, ya que la preocupación creciente por los temas medioambientales se enlaza con la consideración de lo patrimonial como un todo, natural y artificial, que debe ser preservado. La misma característica de todo aquello que

7 Para cualquier consulta sírvase escribir a: pbazzetti13@hotmail.com 
tiene relación con el mar, un autentico mundo en sí mismo, ha contribuido a la creación de este concepto. Pero, para la persona ajena a las actividades del mar se trata de una cultura que puede parecer muy extraña.

Forma parte del patrimonio marítimo muchos elementos diferentes. La lista puede ser enorme, pero el denominador común es siempre el valor del objeto como testimonio de una actividad marítima. Lógicamente también hay que incluir en el patrimonio marítimo todos los vestigios de la historia marítima que se encuentran sumergidos (naufragios). Definitivamente, el patrimonio marítimo es un todo, pese a la tendencia de algunos investigadores a dividir los conocimientos. Eso es un error, ya que pocas cosas hechas por el hombre pueden ser estudiadas aisladamente, fuera de contexto.

En primer lugar, están las embarcaciones, desde el navío más grande a la más pequeña de las embarcaciones. Una nave es el reflejo de lo que una sociedad es capaz de hacer para adaptarse a su naturaleza y llevar a cabo una actividad específica. Un navío o un acorazado, pueden llegar a ser una de las máquinas más complejas que pueda construir un país, y como tal, testimonio muy rico para comprender un momento en la historia de un pueblo, por lo que no es de extrañar que se diga que "aquel país que es capaz de construir un buque de quilla a perilla sea una nación desarrollada".

Igualmente importantes, si no más, son todos los documentos referentes a la historia y a las actividades marítimas, desde un contrato de construcción naval hasta un registro de embarque que nos lleva a un cargamento concreto. Para el historiador puede ser tan útil una crónica virreinal, así como el libro de cuentas de un comerciante, de forma que no hay que valorar la importancia de un documento en función de su aparente importancia. Además, muchos documentos están hermosamente complementados con grabados que también aportan información de valor.

También son patrimonio marítimo las estructuras arquitectónicas e industriales que tienen que ver con la navegación y las actividades marítimas, y que son muestra de la arquitectura y la ingeniería de cada nación y cada época: puertos, faros, almacenes, máquinas, etc. El desarrollo de la arqueología industrial en los últimos años ha conseguido focalizar el interés de muchos estudiosos y del gran público por este tipo de elementos. Relacionados con la técnica están también los instrumentos de navegación, las artes de pesca, las herramientas, etc. En cada uno de ellos hay a veces siglos de avances y retroceso.

Naturalmente todas las manifestaciones del arte que tienen que ver con el mar, o que encuentran en él su fuente de inspiración, pueden ser consideradas patrimonio marítimo. En pintura, incluso puede hablase de especialidades como las marinas (paisajes marítimos o costeros) o el retrato del buque en sí mismo. La escultura, la literatura, la música, las artes decorativas, la fotografía, todas 
tienen su lugar. También el modelismo de barcos, en todas sus formas, puede llegar a convertirse en objetos artísticos, tal como los que encontramos en los museos.

Finalmente hay algunos elementos de difícil clasificación, pero que pueden encerrar en sí mismos también la esencia de la cultura marítima: el léxico la toponimia, la gastronomía, etc. Un elemento fundamental es el patrimonio inmaterial marítimo; el conjunto de sabidurías y conocimientos acumulado durante miles de años por capitanes, marineros, carpinteros, etc. En este mismo grupo se incluyen tradiciones, supersticiones, creencias, leyendas, y cualquier otro elemento intelectual como la música, el vocabulario, las fiestas, etc. Pero por ser la gente de mar, poco dada la literatura en general, apenas han quedado testimonios escritos de muchos de estos saberes, y aunque ha habido investigadores que han recogido cuanto han podido, seguramente es mucho más lo que se ha perdido para siempre.

El patrimonio marítimo está amenazado por serios peligros en todo el mundo, pudiendo en pocos años desaparecer muchos testimonios que nos recuerdan aún miles de años de historia de la navegación. Sin embargo, en pocas décadas los cambios han sido brutales como en el siglo XX. Además de las guerras, el robo, el tráfico de arte y antigüedades y otros enemigos naturales del patrimonio cultural son peligros que inciden especialmente sobre el patrimonio marítimo.

La desaparición de muchos oficios conlleva la pérdida de técnicas ancestrales y de un vocabulario propio. Las costas de casi todo el mundo están amenazadas por estructuras industriales o por la urbanización, con la pérdida de espacios y actividades antes dedicadas a labores marítimas. La transformación económica en el mundo de la navegación y de la pesca ha provocado la desaparición, en pocos años, de embarcaciones y artes de pesca que poco habían cambiado durante siglos. Junto a esto la revolución tecnológica ha enviado al cajón de los recuerdos infinidad de técnicas e instrumentos hasta hace poco imprescindibles. El mundo cambia a gran velocidad y todo un pasado ligado al mar puede desaparecer sin dejar rastro, empobreciendo nuestro acervo cultural y con él nuestras vidas.

Ante esto disponemos de algunas posibilidades de acción y herramientas. En primer lugar hay que señalar el importante papel de los museos marítimos como guardianes del patrimonio y de la memoria. A su lado están las instituciones y asociaciones relacionadas al mar. Se trabaja en la conservación del patrimonio y en su difusión, pero cada día más desde un punto de vista activo. Un ejemplo de dinamismo pude ser la realización de inventarios de lo que todavía existe y debe ser preservado.

El patrimonio debe ser accesible a la comunidad. Es más, ha de ser algo dinámico, vivo, que no se conserva porque sí, sino que se preserva como un elemento útil 
para las futuras generaciones. Afortunadamente ésta es la tendencia actual en el mundo, y la única que puede garantizar que las personas sientan interés por la cultura, la historia y la tradición. En relación con este último punto, también es importante conseguir que el patrimonio permanezca, siempre que sea posible, en su lugar original, relacionado con su medio y con la población que lo ha generado. Así resulta más sencillo darlo a conocer, sensibilizar e involucrar a la comunidad sobre la importancia de su conservación.

Tenemos dos maneras de proteger el patrimonio marítimo, una es a través de los buques museo. Un buque museo es aquel que ha sido conservado después de su vida activa, reacondicionado como museo es abierto al público con fines educativos, conmemorativos o turísticos. Algunas de estas naves aún pueden navegar por sus propios medios. Existen varios centenares de buques museo a nivel mundial, de diverso tipo y antigüedad, estando algunos de ellos asociados a museos navales que complementan la exposición.

La mayoría de los buques dados de baja acaban desguazados y vendidos como chatarra; o también en el caso de las naves militares suelen ser hundidas en prácticas de tiro real. Sólo unos pocos buques acaban siendo preservados por su importancia o singularidad histórica, normalmente tras la presión de asociaciones privadas, voluntarios, entusiastas y antiguas tripulaciones. El proceso de reconversión de un buque museo suele ser largo y costoso, técnicamente y económicamente.

La otra a través de los museos marítimos, que reúnen, conservan, estudian, interpretan y exhiben objetos y materiales relacionados con el pasado marítimo de la humanidad. Son los principales, aunque no los únicos, depositarios de una parte de la memoria colectiva de los hombres de mar de todos los tiempos y todos los rincones del planeta. Todavía hay muchos elementos patrimoniales de valor en manos de particulares, pero lo cierto es que día a día se pierden para siempre objetos o documentos irremplazables. Por fortuna siempre quedan los museos, que en la medida de sus posibilidades intentan salvar para el futuro el máximo del patrimonio.

En un próximo artículo revisaremos el papel de los buques museo, así como de los museos marítimos y veremos los principales exponentes, tanto en nuestro país como en la región. 


\section{REFERENCIAS BIBLIOGRÁFICAS / WEBGRAFÍA}

1. Busquets, Camil, Cabal, Rafael, Campanera, Albert y García, Enric. (2005), El hombre y la mar. Madrid: Aqualarga Grupo Cultural S.A.

2. Jafari, Jafari. (2000) "Enciclopedia del Turismo". Madrid: Editorial Síntesis S.A.

3. Rubio-Ardanaz, Juan Antonio. (2009): El concepto de patrimonio marítimo y de cultura: gestión museística e iconos públicos en el País Vasco", Itsas Memoria. Revista de Estudios Marítimos del País Vasco, 6, Untzi Museoa-Museo Naval, Donostia-San Sebastián, 57-82 páginas.

4. www.patrimoniomaritimo.cl

5. www.untzimuseoa.eus

6. www.pmaritimomurcia.com 
